\title{
Laser-Processing of $\mathrm{VO}_{2}$ Thin Films Synthesized by Polymer-Assisted- Deposition
}

\author{
Eric Breckenfeld ${ }^{1}$, Heungsoo $\mathrm{Kim}^{2}{ }^{*}$ Edward P. Gorzkowski ${ }^{2}$, Thomas E. Sutto ${ }^{2}$, \\ Alberto Piqué ${ }^{2}$ \\ ${ }^{1}$ National Research Council Fellow at the Naval Research Laboratory, Washington DC 20375, \\ USA \\ ${ }^{2}$ Materials Science and Technology Division, Naval Research Laboratory, Washington, DC \\ 20375, USA \\ *Heungsoo.kim@nrl.navy.mil
}

\begin{abstract}
We investigate a novel route for synthesis and laser-sintering of $\mathrm{VO}_{2}$ thin films via solution-based polymer-assisted-deposition (PAD). By replacing the traditional solvent for PAD (water) with propylene glycol, we are able to control the viscosity and improve the environmental stability of the precursor. The solution stability and ability to control the viscosity makes for an ideal solution to pattern simple or complex shapes via direct-write methods. We demonstrate the potential of our precursor for printing applications by combining PAD with laser induced forward transfer (LIFT). We also demonstrate large-area film synthesis on 4 inch diameter glass wafers. By varying the annealing temperature, we identify the optimal synthesis conditions, obtaining optical transmittance changes of $60 \%$ at a $2500 \mathrm{~nm}$ wavelength and a two-order-of-magnitude semiconductor-to-metal transition. We go on to demonstrate two routes for improved semiconductor-to-metal characteristics. The first method uses a multi-coating process to produce denser films with large particles. The second method uses a pulsed-UV-laser sintering step in films annealed at low temperatures $\left(<450^{\circ} \mathrm{C}\right)$ to promote particle growth and improve the semiconductor-to-metal transition. By comparing the hysteresis width and semiconductor-to-metal transition magnitude in these samples, we demonstrate that both improvement methods yield high quality $\mathrm{VO}_{2}$ with a three-order-ofmagnitude transition.
\end{abstract}

Keywords: polymer-assisted-deposition, vanadium dioxide, laser sintering, semiconductormetal transition 


\section{Introduction}

Transition-metal oxides have attracted considerable attention from researchers over the past three decades. One in particular, vanadium dioxide $\left(\mathrm{VO}_{2}\right)$, has emerged as one of the most widely studied functional oxide materials due to its temperature-dependent, first-order semiconductor-to-metal transition ${ }^{[1]}$ which is responsible for a very distinctive set of optical and electrical responses. ${ }^{[2,3]}$ A number of works have demonstrated the ability to tune the transition with strain, ${ }^{[4]}$ chemical doping, ${ }^{[5,6]}$ and ionic gating. ${ }^{[7]}$ Others have suggested potential applications in resistive switching, optical storage, light modulators, thermochromic smart coatings and displays to name a few. ${ }^{[8-11]}$ Synthesis of $\mathrm{VO}_{2}$ thin films has been accomplished by various techniques including physical vapor deposition (molecular beam epitaxy, ${ }^{[12]}$ sputtering, ${ }^{[13]}$ pulsed laser deposition $\left.{ }^{[14]}\right)$, chemical vapor deposition, ${ }^{[15]}$ and electrodeposition. ${ }^{[16]}$ High-quality films have been obtained in all cases, but many of these processes are associated with high capital costs and small coating areas (for physical vapor deposition). ${ }^{[17]}$ Furthermore, conformal deposition on irregular surfaces can be difficult. ${ }^{[18]}$ More recently, scientists have begun exploring solution-based chemical synthesis processes, beginning with the sol-gel process, which is often heralded as inexpensive and scalable. ${ }^{[19]}$ However, careful environmental control is highly important and often difficult for the reactive organometallic precursors which sol-gel requires. Indeed, precursor-water reactions can easily form metal-oxides or hydroxides and lead to precipitates within the organometallic solution, precluding the synthesis of uniform, single-phase films. ${ }^{[20]}$ Other solution-based processes include the hydrothermal ${ }^{[21,22]}$ and hot colloidal ${ }^{[23]}$ synthesis methods. Overall, solution synthesis methods are advantageous due to their potential for low-cost, flexible, reproducible, and scalable fabrication on a variety of substrates. Furthermore, when the precursor solutions are highly stable in ambient conditions, scalable patterning techniques such as inkjet printing or laser-direct-writing are possible. Toward this, an aqueous, polymer-stabilized chemical 
solution process was developed which relies on covalent bonding between aqueous metal ions and functional polymers. ${ }^{[24-26]}$ This process, referred to as polymer-assisted deposition (PAD), provides several advantages. First, the metal-polymer precursor solution is relatively stable, with a shelf-life on the order of months. Second, almost any metal ion can be bound to the functional polymer, without the limitations of organometallic chemistry present in the sol-gel process. Third, the viscosity of the precursor solution, a crucial parameter for patterning and printing, is easily adjustable based on the solvent to polymer ratio.

Several prior works have used the PAD process to synthesize $\mathrm{VO}_{2}$ films. ${ }^{[27-30]}$ While overall successful at obtaining the desired phase, many require reagent materials which are expensive and toxic $\left(\mathrm{VOCl}_{2}\right.$, for example), utilizing complex, multi-step processes for synthesizing the precursor solution. Others require high temperatures $\left(750^{\circ} \mathrm{C}\right)$ and reducing gas $\left(\mathrm{H}_{2}\right)$ during the annealing steps. ${ }^{[31]}$ Furthermore, while these prior works report large changes in optical transmittance between the metallic and insulating states, they generally suffer from a wide temperature hysteresis (at least $30^{\circ} \mathrm{C}$ ) and neglect to report the electrical transport behavior. Here, we demonstrate a simple route to synthesizing a $\mathrm{VO}_{2} \mathrm{PAD}$ precursor solution using common, off-the-shelf, commercially available reagents. By replacing the water solvent with propylene glycol, we demonstrate a new method for controlling precursor viscosity and concentration, which enables the growth of $\mathrm{VO}_{2}$ films with highly reproducible thicknesses and properties and allows for printing of complex shapes through various directwrite techniques. Using this method, we are able to fabricate relatively thick $(\sim 150 \mathrm{~nm})$, continuous $\mathrm{VO}_{2}$ films for which we provide structural, optical, and electrical characterization. We note that our synthesis process utilizes relatively low temperatures $\left(<500{ }^{\circ} \mathrm{C}\right)$ and simple $\mathrm{N}_{2}$ annealing gas. We demonstrate large area film synthesis (4 inch diameter wafer) and the capability to print complex patterns via LIFT. Finally, we establish two routes for improving the transport characteristics of the $\mathrm{PAD} \mathrm{VO}_{2}$ films: UV laser sintering to increase particle size and a multi-coating deposition to improve film density. Indeed, one of the reasons that LIFT 
was selected as a direct write technique was to explore both laser-based patterning and laser annealing with the same system. Both routes for improving the transport characteristics were seen to increase the magnitude of the films semiconductor-to-metal transition to three ordersof-magnitude and reduce the width of the hysteresis below $20^{\circ} \mathrm{C}$. The result of these improvements is the large area synthesis by $\mathrm{PAD}$ of $\mathrm{VO}_{2}$ films with properties nearly matching those of the best quality $\mathrm{VO}_{2}$ grown by vapor or solution deposition techniques.

\section{Material and methods}

The precursor solution was synthesized by mixing 1.5 grams of ammonium metavanadate $\left(\mathrm{NH}_{4} \mathrm{VO}_{3}\right.$, analytically pure), 6.0 g EDTA (ethylenediaminetetraacetic acid), and $3.0 \mathrm{~g}$ PEG (polyethylene glycol, molecular weight 20,000) together in $30 \mathrm{~mL}$ deionized $\mathrm{H}_{2} \mathrm{O}$ (Figure 1a). The solution was stirred at $40^{\circ} \mathrm{C}$ for 12 hours until a blue solution formed. Large, undissolved particles of EDTA were then filtered out. The color of the precursor approximately indicates the oxidation state of the vanadium in-solution, and depends upon the ratio of EDTA to $\mathrm{NH}_{4-}$ $\mathrm{VO}_{3}$ (Figure 1b). When the ratio of EDTA: $\mathrm{NH}_{4} \mathrm{VO}_{3}$ reagents is 2:1, a yellow solution forms, indicating a +5 oxidation state. When the ratio is $4: 1$, a blue solution forms, corresponding to a +4 oxidation state. Finally, when the ratio is $5: 1$, a green solution forms, which corresponds to a +3 oxidation state. For this study, we exclusively used a precursor with a 4:1 mixture of EDTA: $\mathrm{NH}_{4} \mathrm{VO}_{3}$. In order to increase film viscosity and improve the repeatability of the process, the water was replaced as the solvent with propylene glycol after mixing. This was accomplished by adding propylene glycol to the solution and subsequently holding the solution at $50^{\circ} \mathrm{C}$ for 12 hours until the water had mostly evaporated. The resulting precursor was found to be extremely stable at room temperature, with a shelf life on the order of months, and improved the repeatability between growths (see Figure S1 for data on film growth via water solvent). 
The precursor films were deposited on glass substrates by spin coating at $100 \mathrm{RPM}$ for 5 seconds and 3000 RPM for 30 seconds. Large area deposition on a 4-inch-diameter glass wafer can be seen in Figure 1c. We note that the 4-inch-wafer size was limited by the size of the furnace used to anneal the sample and is not a fundamental limitation of the technique itself. Residual water was removed from the films by drying at $100^{\circ} \mathrm{C}$ in air for 10 minutes. Finally, the samples were annealed at temperatures ranging from $400^{\circ} \mathrm{C}$ to $600^{\circ} \mathrm{C}$ for $2 \mathrm{~h}$ in flowing $99.999 \%$ pure $\mathrm{N}_{2}$ with a heating rate of $5^{\circ} \mathrm{C} / \mathrm{min}$. Inert $\mathrm{N}_{2}$ is used to preserve the +4 oxidation state of the vanadium within the solution. Prior studies on the PAD process indicate that the polymer is generally $100 \%$ decomposed by $400-500^{\circ} \mathrm{C},{ }^{[32]}$ with full oxidation and crystallization of the film obtained at slightly higher temperatures. Polymer decomposition is confirmed via thermogravimetric analysis in Supplemental Materials section 2. We confirm that roughly $2.5 \%$ of the mass of the original solution remains after the sample reaches 375 $400^{\circ} \mathrm{C}$ (Figure S2).

In between the spin-coating and annealing steps, it is also possible to pattern complex shapes from the precursor solution using the LIFT process. LIFT is a non-contact, nozzle-free additive manufacturing process capable of printing a variety of fluids. In the LIFT process, small fluid droplets are transferred from a donor substrate to a receiver substrate using a pulsed laser. ${ }^{[33,34]}$ For this work, a $355 \mathrm{~nm}$ laser is used. This is shown schematically in Figure 1a (bottom 3 panels), where the spin-coated substrate is used as the donor and a separate glass substrate is used as the receiver. Details of the LIFT are provided in the Supplemental Materials section 3. Using LIFT, we patterned a $\mathrm{VO}_{2}$ thin film into the letters, "NRL" (Figure 1d), demonstrating that the process can be used to print any arbitrary shapes.

$\mathrm{X}$-ray diffraction (XRD) [Rigaku rotating anode X-ray generator with $\mathrm{Cu} \mathrm{K}_{\alpha}$ radiation] was used to characterize the crystal structure of the films. The optical transmittance spectra were measured in air using a UV-visible-near-IR spectrophotometer [JASCO, V-670]. The electrical switching properties of the $\mathrm{VO}_{2}$ films were characterized at temperatures 
between 25 and $110{ }^{\circ} \mathrm{C}$ by a linear four-probe method using a current of $20 \mu \mathrm{A}$. Film thickness was determined with a KLA tencor P-15 stylus profilometer.Laser sintering was performed by exposing the films to a number of pulses from a $248 \mathrm{~nm}$ UV excimer laser with a $20 \mathrm{~ns}$ pulse duration. The laser beam passes through a lens with a $300 \mathrm{~mm}$ focal length and a $12.5 \mathrm{~mm}$ diameter, circular iris, after which point it is incident upon the sample. Laser fluence, which is defined as the energy per unit area per pulse, was determined by measuring the energy of the laser using a calibrated, external energy monitor. Optimization of operating voltages was used to assure minimal laser fluence deviation between pulses. The laser spot size was measured using laser thermal paper. For this study, a laser energy of $51 \mathrm{~mJ}$ per pulse and a spot size of $1.28 \mathrm{~cm}^{2}$ were used, resulting in a fluence of $\sim 40 \mathrm{~mJ} / \mathrm{cm}^{2}$.

\section{Results and Discussion}

$\mathrm{XRD}$ and optical studies were conducted to determine which annealing temperatures are best suited for synthesis of $\mathrm{VO}_{2}$. As noted in the previous section, films were annealed at temperatures ranging from $400-600^{\circ} \mathrm{C}$. Optimal annealing temperatures were determined based on analysis of these XRD patterns. For the sample annealed at $440^{\circ} \mathrm{C}$ (Figure 2a blue), no peaks are observed. This could indicate either that the film is amorphous or that the presence of any crystalline phases is below our detection limits. As the annealing temperature is increased to $500^{\circ} \mathrm{C}$ and $550^{\circ} \mathrm{C}$, various peaks corresponding to polycrystalline $\mathrm{M}-\mathrm{VO}_{2}$ appear (Figure 2a, black and red). No peaks can be detected for other vanadium oxides such as $\mathrm{V}_{2} \mathrm{O}_{5}$ or $\mathrm{V}_{2} \mathrm{O}_{3}$. Based on these results, we conclude that our films annealed between $500^{\circ} \mathrm{C}$ and $550^{\circ} \mathrm{C}$ are primarily monoclinic polycrystalline $\mathrm{VO}_{2}$, with the presence of any parasitic phases below our detection limits. Moving along, optical studies reveal semiconductor-to-

metal transitions for all annealing temperatures between $400^{\circ} \mathrm{C}$ and $550^{\circ} \mathrm{C}$. For the film annealed at $440^{\circ} \mathrm{C}$ (Figure $2 \mathrm{~b}$, blue), the room temperature (semiconductor state) optical transmittance is $87 \%$ and the high temperature (metallic state) optical transmittance is $51 \%$ at 
a wavelength of $2500 \mathrm{~nm}$, corresponding to a total $\Delta$ transmittance of $36 \%$. This seems to indicate that the film is crystalline $\mathrm{VO}_{2}$ and not amorphous despite difficulty identifying clear peaks in XRD due to the small particle size. Next, for the film annealed at $500^{\circ} \mathrm{C}$ (Figure $2 \mathrm{~b}$, black), the room temperature (semiconductor state) optical transmittance is measured at $86 \%$ and the high temperature (metallic state) optical transmittance is measured at $42 \%$ at a wavelength of $2500 \mathrm{~nm}$, corresponding to a $\Delta$ transmittance of $44 \%$. Finally, for films annealed at $550^{\circ} \mathrm{C}$ (Figure $2 \mathrm{~b}$, red), the room temperature (semiconductor state) optical transmittance is measured at $89 \%$ and the high temperature (metallic state) optical transmittance is measured at $49 \%$ at a wavelength of $2500 \mathrm{~nm}$, corresponding to a $\Delta$ transmittance of $40 \%$. Transmittance plots as a function of temperature for all films annealed between $400^{\circ} \mathrm{C}$ and $550^{\circ} \mathrm{C}$ can be found in Figure S4. Based on the XRD and optical studies, we conclude that annealing at $500-550^{\circ} \mathrm{C}$ yields the highest quality $\mathrm{VO}_{2}$ films. Lower temperature annealing, while still yielding films with a clear semiconductor-to-metal transition, results in poorer crystallinity (from XRD) and a diminished optical transition.

Unlike most prior works on the PAD synthesis of $\mathrm{VO}_{2}$ films, reported here are the electrical transport characteristics for three $80 \mathrm{~nm} \mathrm{VO}_{2}$ films on glass. ${ }^{[27-30]}$ The absence of electrical transport data in prior works could potentially be due to the fact that these studies wished to focus more on the optical responses and thermochromic properties of these films or that it was difficult to synthesize continuous films at thicknesses below $100 \mathrm{~nm}$. Here, electrical transport as a function of temperature was measured in a four-point collinear configuration in order to determine the magnitude and character of the semiconductor-tometal transition. Results for films annealed at $440^{\circ} \mathrm{C}$ (Figure 2c, blue) and $500^{\circ} \mathrm{C}$ (Figure 2c, black) are provided. It is important to note here that some films could not be reliably measured. Films annealed at $550^{\circ} \mathrm{C}$ or higher proved to be electrically insulating and are confirmed by SEM and AFM (in the next section) to be discontinuous. The films annealed at $440^{\circ} \mathrm{C}$ and $500^{\circ} \mathrm{C}$ all exhibit relatively sharp semiconductor-to-metal transitions spanning 
roughly two orders of magnitude. As the annealing temperature is increased, the magnitude of the electrical transition increases slightly. Additionally, the onset of the transition moves from $50-55^{\circ} \mathrm{C}$ for the film annealed at $440^{\circ} \mathrm{C}$ to $65-70^{\circ} \mathrm{C}$ for the film annealed at $500^{\circ} \mathrm{C}$. Finally, the width of the hysteresis decreases somewhat as annealing temperature is increased. While these results can be partially explained based on the improved crystallinity associated with higher annealing temperatures, SEM and AFM morphology studies are required to provide a full picture.

SEM studies for films annealed at $440^{\circ} \mathrm{C}, 500^{\circ} \mathrm{C}$, and $550^{\circ} \mathrm{C}$ (Figure $2 \mathrm{~d}-\mathrm{f}$ ) show a clear trend of increasing particle size with increasing annealing temperature. AFM images for several films annealed between $440^{\circ} \mathrm{C}$ and $600^{\circ} \mathrm{C}$ confirm this trend (Figure 3a-f). This is most likely attributed to higher mobility of growth species on the substrate surface. We can now explain a number of trends based on the SEM and AFM data. First, the large particle sizes observed in samples annealed at $550^{\circ} \mathrm{C}$ ultimately yield discontinuous films. This explains why such samples appeared electrically insulating despite showing the highest crystallinity in XRD and a large optical transition between room temperature and high temperature states. Second, increasing particle size reduces particle boundary scattering, which improves the conductance of the metallic state and thus increases the quality and magnitude of the electrical transition. Third, as expected, surface roughness was found to increase with increased annealing temperature (Figure 3g). Overall, it is clear that large particle sizes are desirable for maximizing the performance of the $\mathrm{VO}_{2}$ films. However, the decrease in connectivity between particles, resulting from their growth at high annealing temperatures, potentially limits the use of these films for electronic applications. Toward this, we conclude by exploring two new routes for yielding continuous PAD films with improved particle density.

The first method for growth of continuous films with improved particle density is a multi-step deposition process using a dilute precursor solution. In this method, a second 
coating of polymer precursor solution is applied after the first annealing step and the subsequent film is placed back into the furnace and annealed at the same temperature. The goal is to infiltrate the porous structure of the film with an additional layer of precursor solution and fill in the gaps between crystallites. This process is performed between one and three times, resulting in two-layer and four-layer samples. The concentration of the solution was adjusted to ensure that the final thickness of each film was roughly similar. Thus, the solution for single-layer films was synthesized by mixing propylene glycol and the aqueous precursor solution at a mass ratio of 1:2. The solution for double-layer films was mixed at a ratio of 1:1, and the solution for four-layer films was mixed at a ratio of $2: 1$. In all cases, the resulting films (1 layer at 2:1, 2 layers at 1:1, or four layers at 1:2) were $\sim 150 \mathrm{~nm}$ thick. AFM (Figure 4a, b, and c) and electrical data (Figure 4g) are provided for a single-layer, doublelayer, and four-layer films. The AFM data shows improved particle density for a double-layer and four-layer film (Figure 4b and c, respectively) compared to a single-layer film (Figure 4a). Electrical transport measurements (Figure 4g) show a gradual improvement in the magnitude of the semiconductor-to-metal transition as the number of layers is increased. Four-layer films possess a roughly three-order-of-magnitude transition, improving upon the single-layer films by a factor of 10 . We also note that discontinuous, single-layer films annealed at higher temperatures $\left(550^{\circ} \mathrm{C}\right.$ or higher) can be made continuous after multiple coatings. Thus, this multiple coating process using adjustable precursor concentrations helps to lower the final film thickness for achieving higher transmittance as well as to reduce the surface roughness, which improves the electrical properties (magnitude of the electrical resistance change). In this study, we did not find the electrical properties of these multi-coating films annealed at $550^{\circ} \mathrm{C}$ to be appreciably different from those annealed at $500^{\circ} \mathrm{C}$. These observations confirm a significant improvement in electrical properties over single-layer samples. Although the high annealing temperature $\left(500^{\circ} \mathrm{C}\right)$ and multiple spin coating steps may be seen as 
disadvantageous, the improvement to the electrical properties demonstrate that this process may have significant merit.

In previous studies on porous oxide networks, UV laser sintering has been demonstrated as a potential method for improving electrical connectivity between particles. ${ }^{[35,36]}$ Laser sintering has also been previously shown to increase particle size in a number of systems including metals and ceramics. ${ }^{[37]}$ For this reason, we conclude by conducting a post-furnace laser sintering step on low annealing temperature samples in order to improve the transport character. We begin with a $150 \mathrm{~nm}$ film annealed at $440^{\circ} \mathrm{C}$ in $\mathrm{N}_{2}$ for 24 hours. The annealing time for this sample was increased from $2 \mathrm{~h}$ to $24 \mathrm{~h}$ to promote higher crystallinity prior to laser sintering and minimize polymer precursor residue. Films were exposed to a $248 \mathrm{~nm}$ UV excimer laser between 1 and 50 times with a laser fluence of $\sim 40$ $\mathrm{mJ} / \mathrm{cm}^{2}$. Details about the optical setup for sintering and determination of laser fluence can be found in the Supplemental Materials section 5. Surface topography as a function of laser pulses can be seen for as-grown films, films exposed to 5 excimer pulses, and films exposed to 50 excimer pulses in Figure $4 \mathrm{~d}, 4 \mathrm{e}$, and $4 \mathrm{f}$, respectively. The mean particle size for each film is provided in the Supplemental Materials Section, Table S1. Note the significant increase in average particle size between the as-grown film (Figure $4 \mathrm{~d}, 50 \mathrm{~nm}$ average particle size) and the film treated 50 times with the sintering laser (Figure 4f, $81 \mathrm{~nm}$ average particle size). Electrical resistivity as a function of temperature is provided for films exposed to 0,5 , 25, and 50 pulses. (Figure 4h). As the number of laser pulses increases, there are three distinct trends. First, the overall resistivity is reduced for both the semiconducting and metallic states. Second, the magnitude of the semiconductor-to-metal transition is increased. Third, the width of the hysteresis decreases from nearly $30^{\circ} \mathrm{C}$ for as-grown films to less than $20^{\circ} \mathrm{C}$ for films exposed 50 times. These improvements can be attributed to increases in particle size as confirmed by AFM analysis. A summary of the properties of the $\mathrm{VO}_{2}$ films obtained in this 
work and their comparison with those made by other techniques as reported in the literature can be found in table $\mathrm{S} 1$.

\section{Conclusions}

We have shown the ability to reliably synthesize polycrystalline $\mathrm{VO}_{2}$ films using simple, commercially available reagents, relatively low annealing temperatures and a nonreactive gas $\left(\mathrm{N}_{2}\right)$ atmosphere by the polymer-assisted-deposition (PAD) process in combination with laser processing. We have determined the best processing conditions via structural, optical, and electrical transport studies. Optical studies at a wavelength of $2500 \mathrm{~nm}$ indicate a difference in transmittance from $86 \%$ in the insulating state to $42 \%$ in the metallic state for films prepared at optimum conditions, and electrical transport studies indicate a three order of magnitude resistivity change. The synthesis of $\mathrm{VO}_{2}$ films via propylene glycol PAD is important for a number of reasons. First, since the film is polycrystalline, it does not depend on epitaxial alignment with the substrate. Second, the best optical and electrical properties were obtained from films synthesized at $500^{\circ} \mathrm{C}$, a much lower temperature than reported in previous studies. Finally, the utilization of $\mathrm{N}_{2}$ simplifies the process significantly compared to some prior studies that used more reactive gases such as $\mathrm{O}_{2}, \mathrm{H}_{2}$ forming gas, or a staggered combination of both at different stages. The combination of these three factors significantly widens our possible choice of substrates, and although these studies primarily used glass as a growth substrate, the same process could be readily adapted to $\mathrm{Si}$ wafers, mica, or other substrates compatible with these annealing temperatures. We have also demonstrated two routes for improving the PAD process: multi-step deposition and laser-sintering. Both methods yielded continuous films with large particle sizes and sharp semiconductor-to-metal transitions with narrow hysteresis. Additionally, the properties of these samples were found to be comparable to samples from previous studies synthesized by vapor deposition or sol-gel 
(see Table S1). Taken all together, we have demonstrated a robust process for fast, reliable synthesis of $\mathrm{VO}_{2}$ films over potentially large areas at relatively low temperatures.

\section{Acknowledgements}

This work was funded by the Office of Naval Research (ONR) through the Naval Research Laboratory Basic Research Program (Grant No. N0001415WX00004).

\section{Additional Information}

Supplementary material accompanies this paper.

\section{References}

[1] F. J. Morin, Oxides which show a metal-to-insulator transition at the Neel temperature, Phys Rev Lett. 3 (1959) 34-36.

[2] T. D. Manning, I. P. Parkin, R. J. H. Clark, D. Sheel, M. E. Pemble, D. Vernadou, Intelligent window coatings: atmospheric pressure chemical vapor deposition of vanadium oxides, J. Mater. Chem 12 (2002) 29362939.

[3] C. G. Granqvist, P. C. Lansaker, N. R. Mlyuka, G. A. Niklasson, E. Avendano, Progress in chromogenics: new results for electrochromic and thermochromic materials and devices, Sol. Energy Mater. Sol. Cells 93 (2009) 2032-2039.

[4] Y. Muraoka, Z. Hiroi, Metal-insulator transition of $\mathrm{VO}_{2}$ thin films grown on $\mathrm{TiO}_{2}(001)$ and (110) substrates Appl. Phys. Lett. 80 (2002) 583-585.

[5] T. Horlin, T. Niklewski, M. Nygren, Electrical and magnetic properties of $\mathrm{V}_{1-\mathrm{x}} \mathrm{W}_{\mathrm{x}} \mathrm{O}_{2}, 0<\mathrm{x}<0.060$, Mater. Res. Bull. 7 (1972) 1515-1524.

[6] K. Shibuya, M. Kawasaki, M. Tokura, Metal-insulator transition in epitaxial $\mathrm{V}_{1-\mathrm{x}} \mathrm{W}_{\mathrm{x}} \mathrm{O}_{2}(0<\mathrm{x}<0.33)$ thin films, Appl. Phys. Lett. 96 (2010) 022102 1-3.

[7] J. Jeong, N. Aetukuri, T. Graf, T.D. Schladt, M.G. Samant, S.S. Parkin, Suppression of metal-insulator transition in $\mathrm{VO}_{2}$ by electric field-induced oxygen vacancy formation, Science 339 (2013) 1402-1405.

[8] M. Gurvitch, S. Luryi, A. Polyakov, A. Shabalov, M. Dudley, G. Wang, S. Ge and V. Yakovlev, VO Vilms $_{2}$ with strong semiconductor to metal phase transition prepared by the precursor oxidation process J. Appl. Phys. 102 (2007) 033504 1-13.

[9] A. Crunteanu, J. Givernaud, J. Leroy, D. Mardivirin, C. Champeaux, J.-C. Orlianges , A. Catherinot and P. Blondy, Voltage- and current-activated metal-insulator transition in $\mathrm{VO}_{2}$-based electrical switches: a lifetime operation analysis, Sci. Technol. Adv. Mater. 11 (2010) 065002 1-6. 
[10] H. Kim, N. Charipar, E. Breckenfeld, A. Rosenberg, A. Piqué, Active terahertz metamaterials based on the phase transition of $\mathrm{VO}_{2}$ thin films, Thin Solid Films $\mathbf{5 9 6}$ (2015) 45-50.

[11] S. Wang, M. Liu, L. Kong, Y. Long, X. Jiang, A. Yu, Recent Progress in $\mathrm{VO}_{2}$ Smart Coatings: Strategies to Improve the Thermochromics Properties. Progress in Materials Science 81 (2016) 1-54.

[12] W. Gao, C. M. Wang, H. Q. Wang, V. E. Henrich, E. I. Altman, Growth and surface structure of vanadium oxide on anatase (0 0 1), Surface Science 559 (2004) 201-213.

[13] I. Balberg, B. Abeles, Y. Arie, Phase transition in reactively co-sputtered films of $\mathrm{VO}_{2} / \mathrm{TiO}_{2}$, Thin Solid Films 24 (1974) 307-310.

[14] H. Kim, N. Charipar, M. Osofsky, S. B. Qadri, A. Piqué, Optimization of the semiconductor-metal transition in $\mathrm{VO}_{2}$ epitaxial thin films as a function of oxygen growth pressure, Appl. Phys. Lett. 104 (2014) $0819131-5$.

[15] B. S. Mathur, T. Ruegamer, I. Grobelsek, Phase-selective CVD of vanadium oxide nanostructures, Chem. Vap. Depos. 13 (2007) 42-47.

[16] J. A. Koza, Z. He, A. S. Miller, J. A. Switzer, Resistance switching in electrodeposited $\mathrm{VO}_{2}$ thin films, Chem. Mater. 23 (2011) 4105-4108.

[17] S. M. Pawar, B. Pawar, J. H. Kim, S. Joo, C. Lokhande, Recent status of chemical bath deposited metal chalcogenide and metal oxide thin films, Curr. Appl. Phys. 11 (2011) 117-161.

[18] D. Hass, Y. Marciano, H. Wadley, Physical vapor deposition on cylindrical substrates, Surf. Coat. Technol. 185 (2004) 283-291.

[19] G. Hodes, Chemical Solution Deposition of Semiconductor Films, Marcel. Dekker, Inc., New York, 2002.

[20] Y. Lin, J.-S. Lee, H. Wang, Y. Li, S. R. Foltyn, Q. X. Jia, G. E. Collis, A. K. Burrell, and T. M. McCleskey, Structural and dielectric properties of epitaxial $\mathrm{Ba}_{1-\mathrm{x}} \mathrm{Sr}_{\mathrm{x}} \mathrm{TiO}_{3}$ films grown on $\mathrm{LaAlO}_{3}$ substrates by polymerassisted deposition, Appl. Phys. Lett. 85 (2004) 5007-5009.

[21] T. Chirayil, P. Y. Zivalij, M. S. Whittingham, Hydrothermal synthesis of vanadium oxides Chem. Mater. 10 (1998) 2629-2640.

[22] D. Alie, L. Gedvilas, Z. Wang, R. Tenent, C. Engtrakul, Y. Yan, S. E. Shaheen, A. C. Dillon, C. Ban, Direct synthesis of thermochromic $\mathrm{VO}_{2}$ through hydrothermal reaction J. Solid State Chem. 212 (2014) 237-241.

[23] T. Paik, S.-H. Hong, E. A. Gaulding, H. Caglayan, T. R. Gordon, N. Engheta, C. R. Kagan, and C. B. Murray, Solution-processed phase-change $\mathrm{VO}_{2}$ metamaterials for colloidal vanadium oxide $\left(\mathrm{VO}_{\mathrm{x}}\right)$ nanocrystals, ACS Nano 8 (2013) 797-806.

[24] Q. X. Jia, T. M. McCleskey, A. K. Burrell, Y. Lin, G. E. Collis, H. Wang, A. D. Q. Li, S. R. Foltyn, Polymer-assisted deposition of metal-oxide films, Nat. Mater. 3 (2004) 529-532.

[25] J. M. Vila-Fungueirino, B. Rivas-Murias, B. Rodriguez-Gonzalez, F. Rivadulla, Interface magnetic coupling in epitaxial bilayers of $\mathrm{La}_{0.92} \mathrm{MnO}_{3} / \mathrm{LaCoO}_{3}$ prepared by polymer-assisted deposition, Chem. Mater. 26 (2014) 1480-1484.

[26] R. E. Jilek, E. Bauer, A. K. Burrell, T. M. McCleskey, Q. Jia, B. L. Scott, M. F. Beaux, T. Durakiewicz, J. J. Joyce, K. D. Rector, J. Xiong, K. Gofryk, F. Ronning, R. L. Martin, Preparation of epitaxial uranium dicarbide thin films by polymer-assisted deposition, Chem. Mater. 25 (2013) 4373-4377.

[27] L. Kang, Y. Gao, H. Luo, A novel solution process for the synthesis of $\mathrm{VO}_{2}$ thin films with excellent thermochromic properties, ACS Appl. Mater. Interfaces 1 (2009) 2211-2218. 
[28] L. Kang, Y. Gao, Z. Zhang, J. Du, C. Cao, Z. Chen, H. Luo, Effects of annealing parameters on optical properties of thermochromic $\mathrm{VO}_{2}$ films prepared in aqueous solution, J. Phys. Chem. C 114 (2010) 1901-1911. [29] J. Du, Y. Gao, H. Luo, L. Kang, Z. Zhang, Z. Chen, C. Cao, Significant changes in phase-transition hysteresis for Ti-doped $\mathrm{VO}_{2}$ films prepared by polymer-assisted deposition, Sol. Energ. Mat. Sol. Cells 95 (2011) 469-475.

[30] F. Yue, W. Huang, Q. Shi, D. Li, Y. Hu, Y, Xiao, X. Deng, C. Wang, Phase transition properties of vanadium oxide films deposited by polymer-assisted deposition, J. Sol-Gel Sci. Technol. 72 (2014) 565-570. [31] Y. Ji, Y. Zhang, M. Gao, Z. Yuan, C. Jin, Y. Lin, Growth and physical properties of vanadium oxide thin films with controllable phases, Mater. Res. Soc. Symp. Proc. 1547 (2013) 21-27.

[32] G. F. Zou, J. Zhao, H. M. Luo, T. M. McCleskey, A. K. Burrelld and Q. X. Jia, Polymer-assisteddeposition: a chemical solution route for a wide range of materials, Chem. Soc. Rev. 42 (2013) 439-449.

[33] S. A. Mathews, R. C. Y. Auyeung, H. Kim, N. Charipar, A. Piqué, High-speed video study of laser-induced forward transfer of silver nano-suspensions, J. Appl. Phys. 114 (2013) 064910.

[34] E. Breckenfeld, H. Kim, R.C.Y. Auyeung, N. Charipar, P. Serra, A. Piqué, Laser-induced forward transfer of silver nanopaste for microwave interconnects, Appl. Surf. Sci. 331 (2015) 254-261.

[35] H. Kim, R.C.Y. Auyeung, M. Ollinger, G.P. Kushto, Z.H. Kafafi, A. Piqué, Laser-sintered mesoporous $\mathrm{TiO}_{2}$ electrodes for dye-sensitized solar cells, Appl. Phys. A. 83 (2006) 73-76.

[36] M. Baum, H. Kim, I. Alexeev, A. Piqué, M. Schmidt, Generation of transparent conductive electrodes by laser consolidation of LIFT printed ITO nanoparticle layers, Appl. Phys. A 111 (2013) 799-805.

[37] Y. P. Kathuria, Microstructuring by selective laser sintering of metallic powder, Surf. Coat. Technol. 116 (1999) 643-647. 

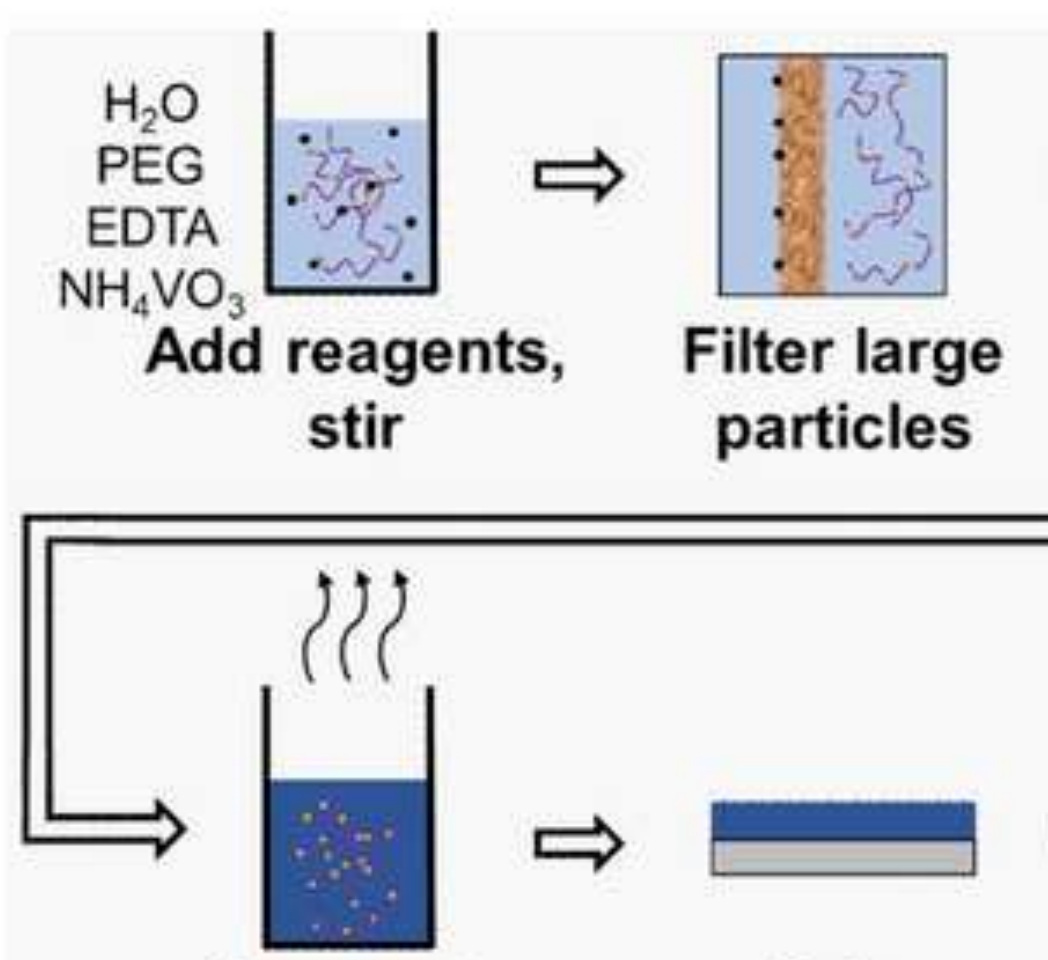

Evaporate

water

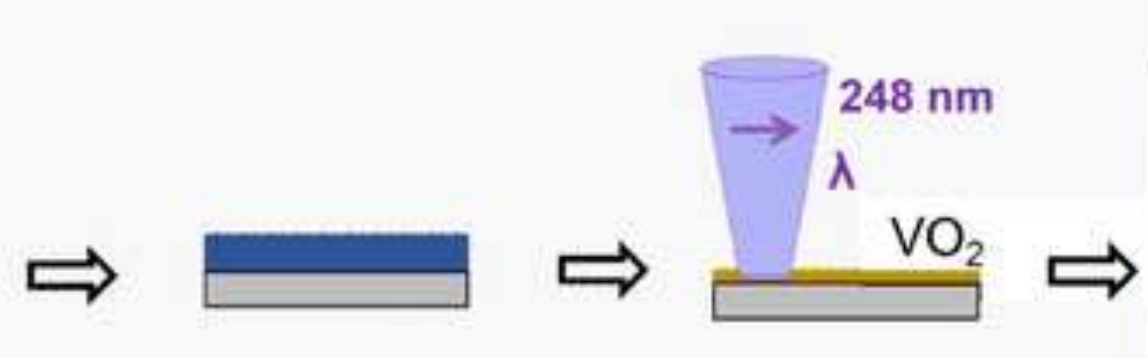

Spin-

coat ink
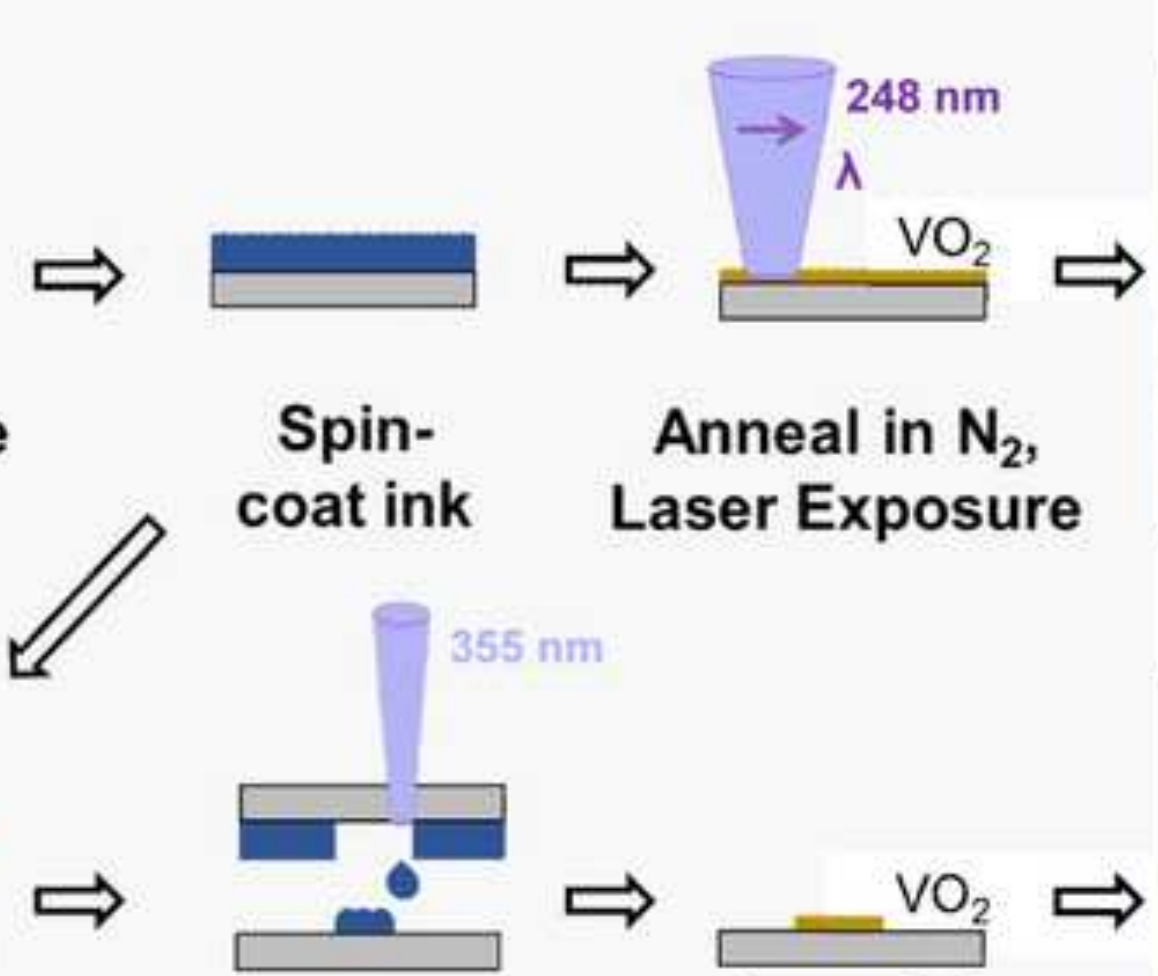

Place above receiver
Laser transfer desired shape

(a)
$\Rightarrow$

Add propylene glycol

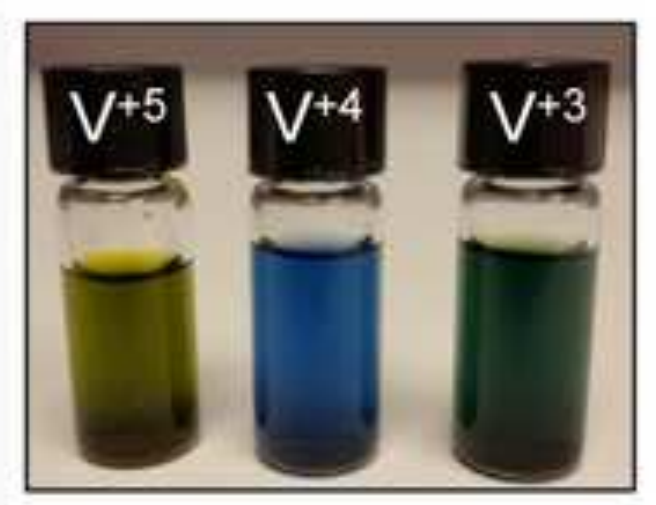

(b)

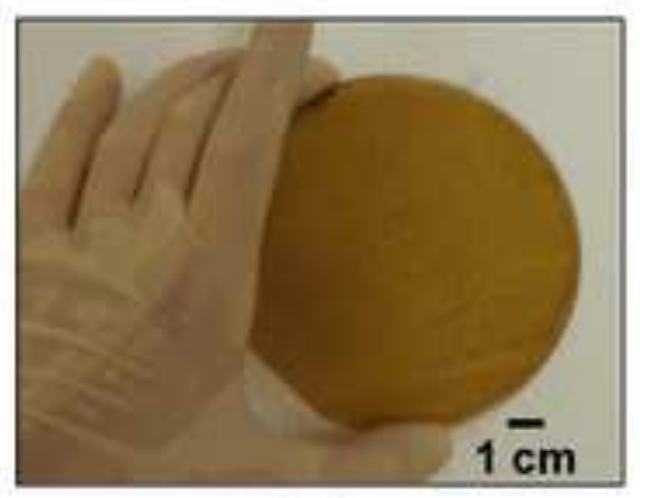

(c)

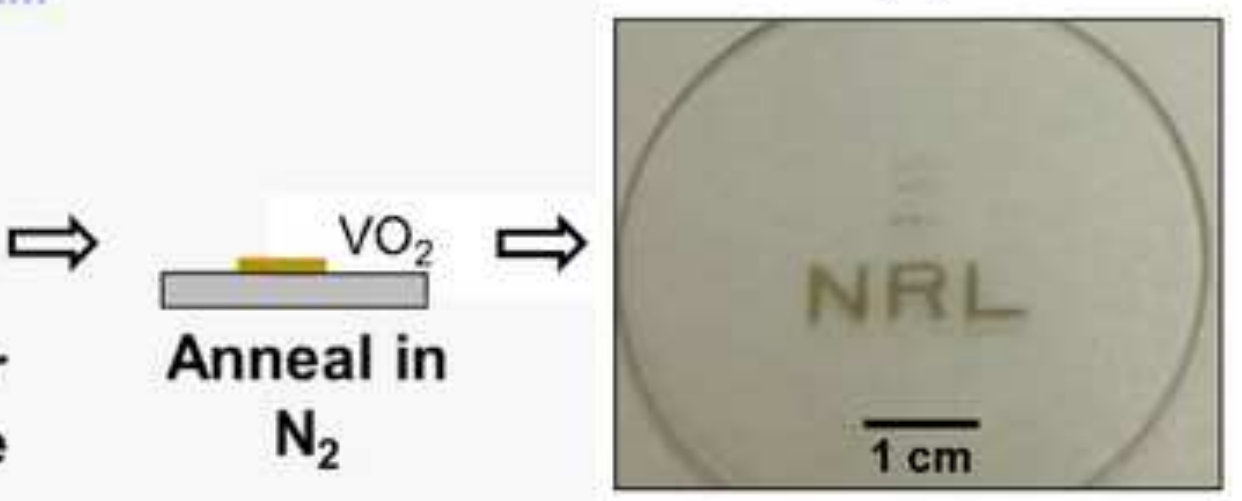

(d) 


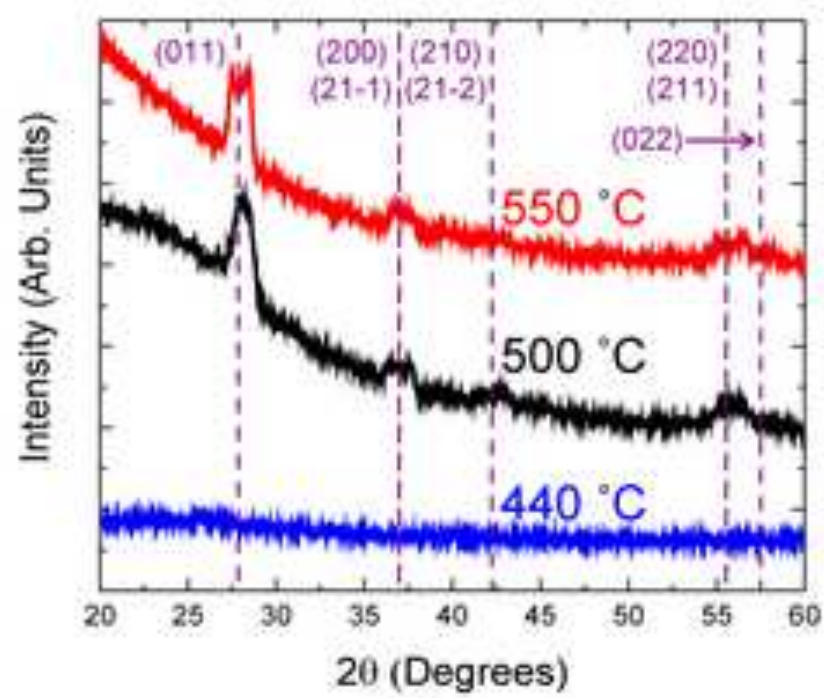

(a)

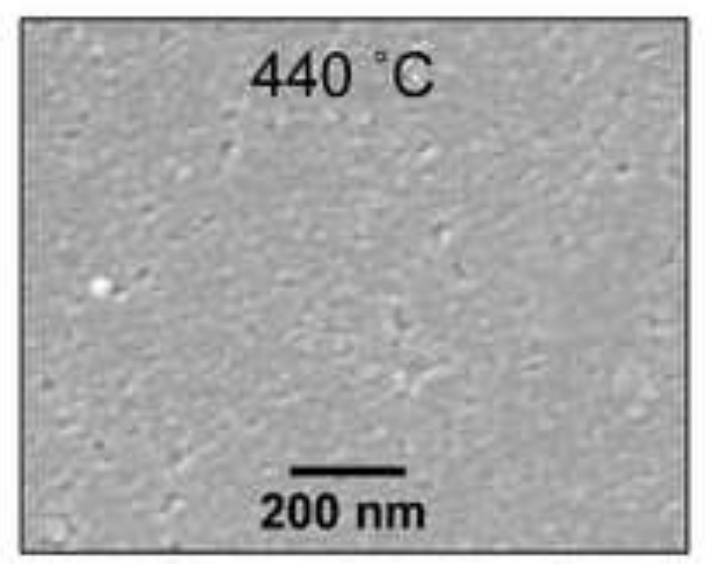

(d)

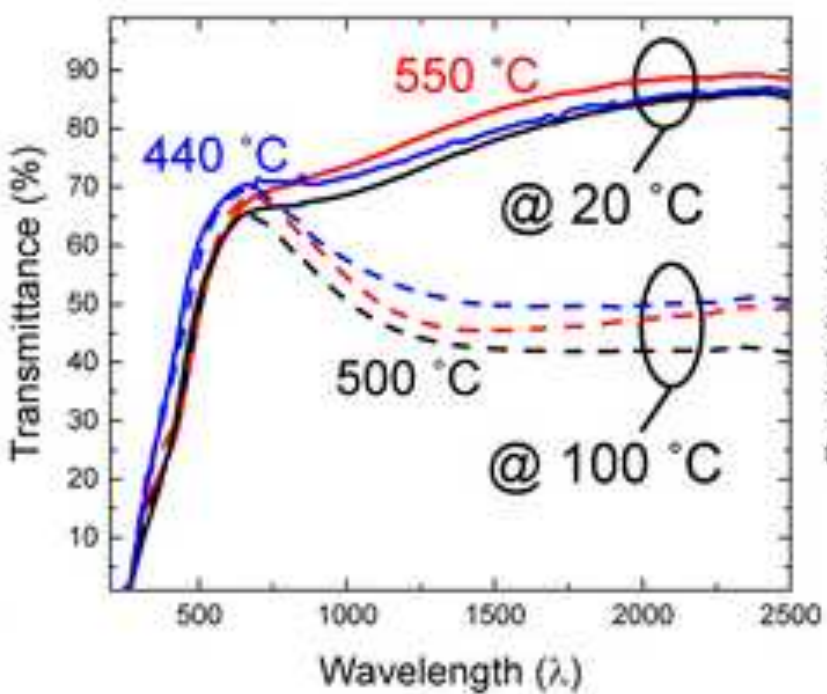

(b)

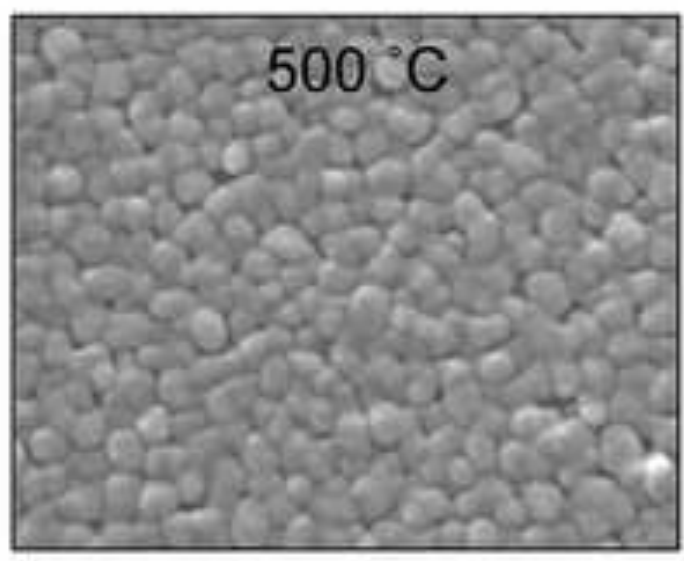

(e)

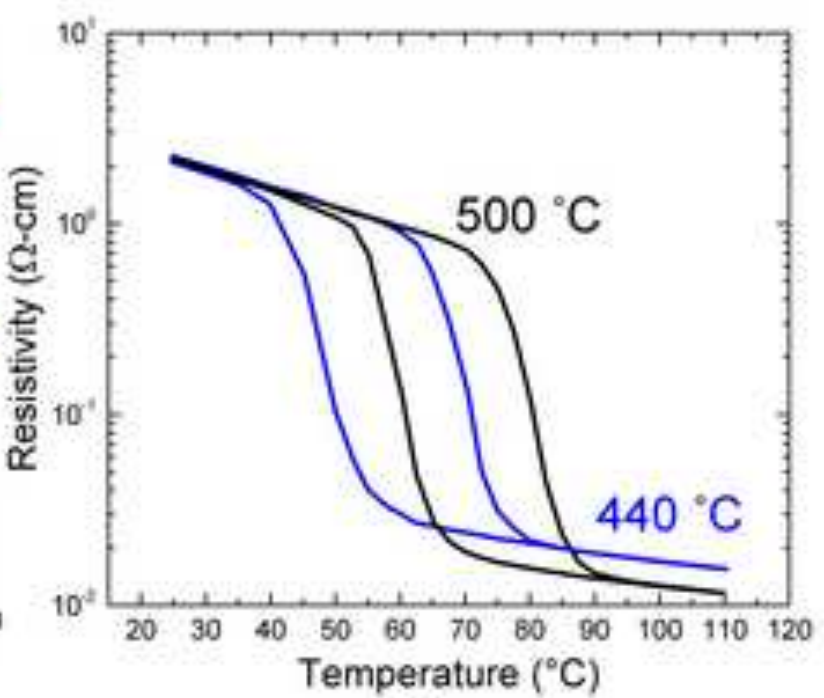

(c)

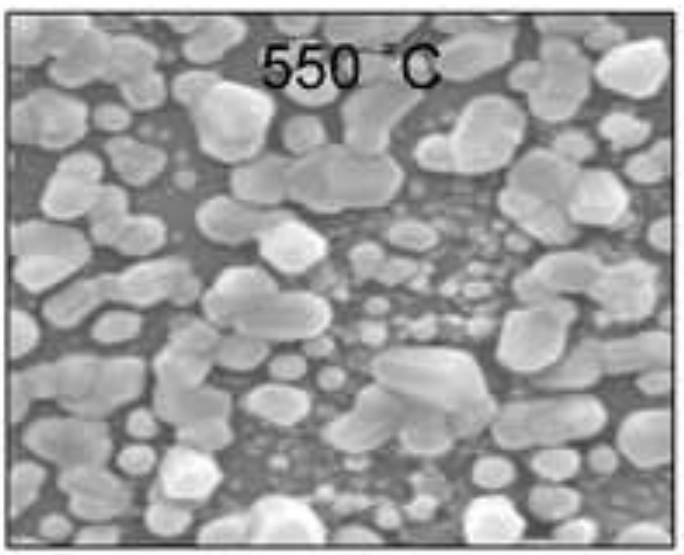

(f) 

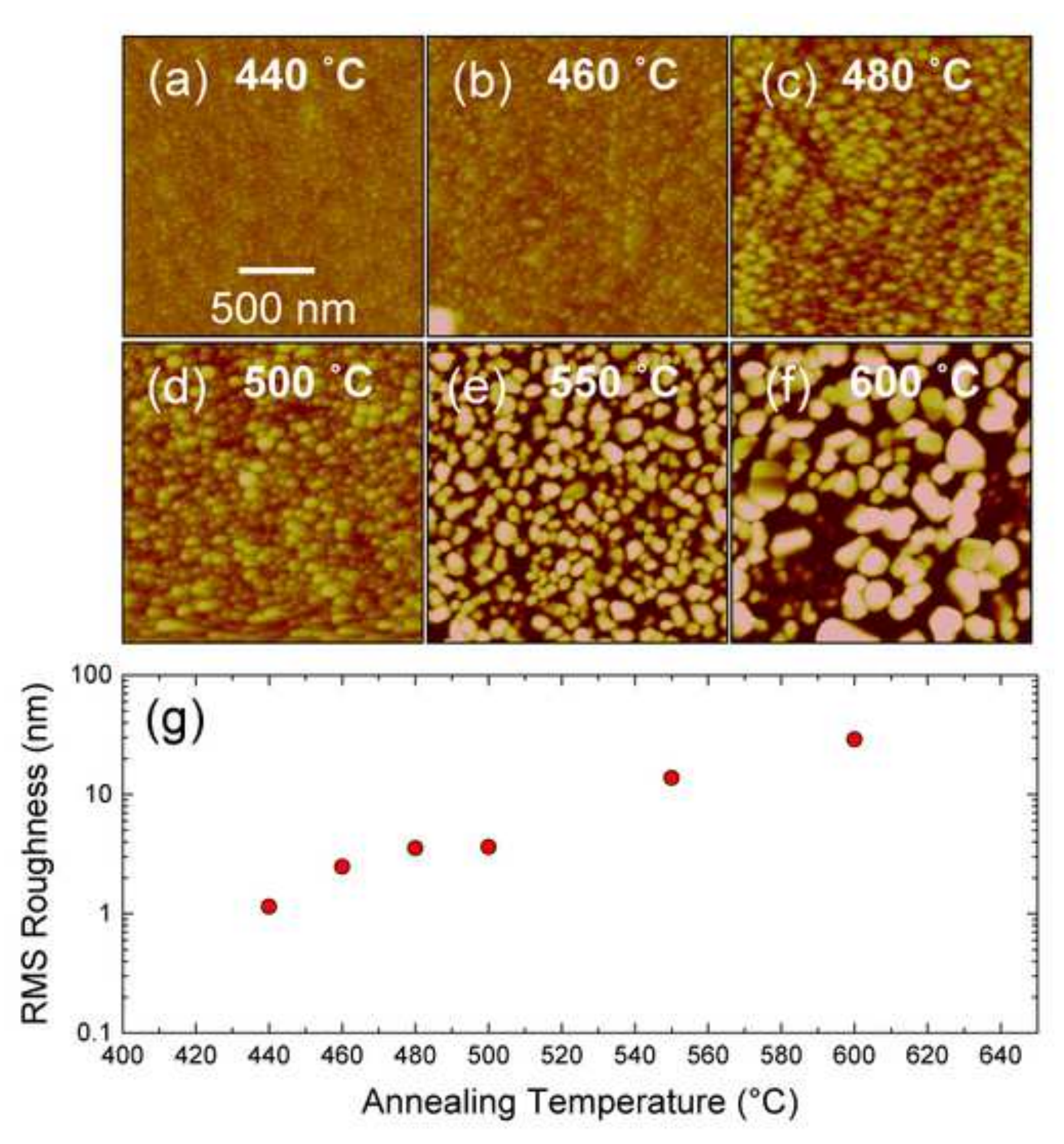

3



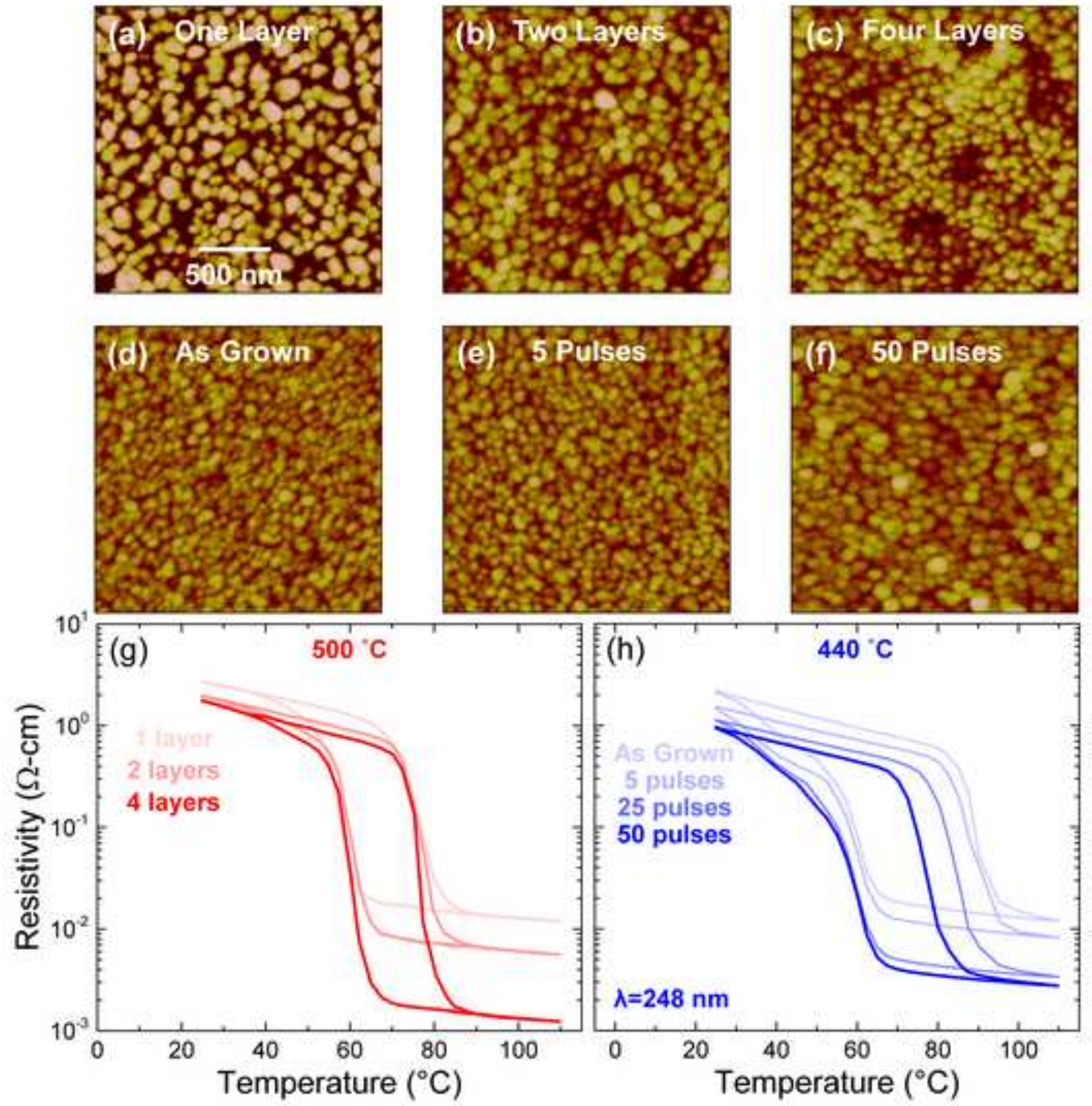

(h) 


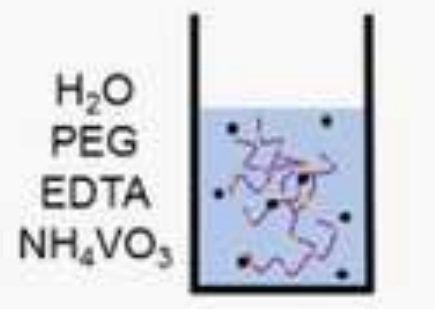

Add reagents, stir

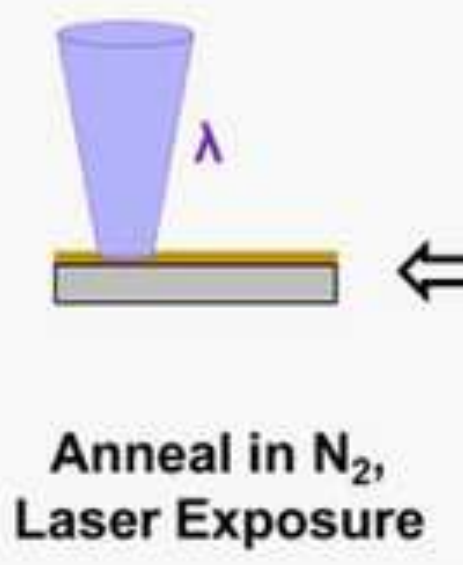

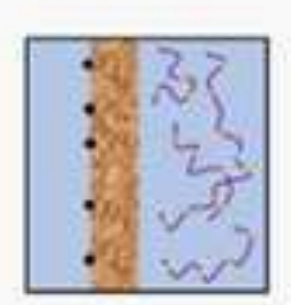

Filter large particles

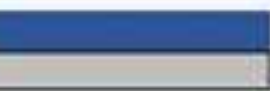

Spin-coat ink

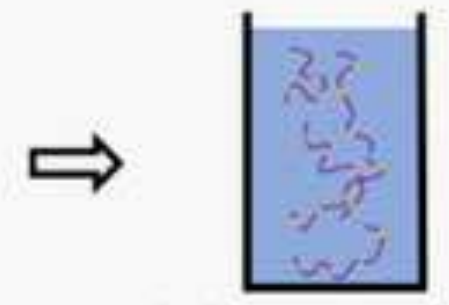

Add propylene glycol

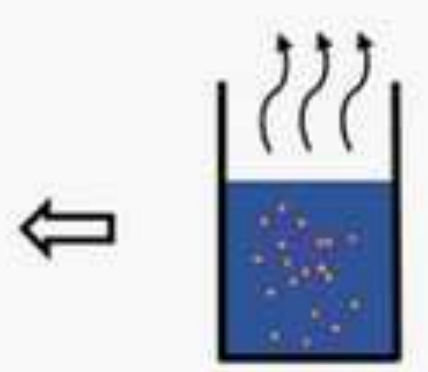

Evaporate water

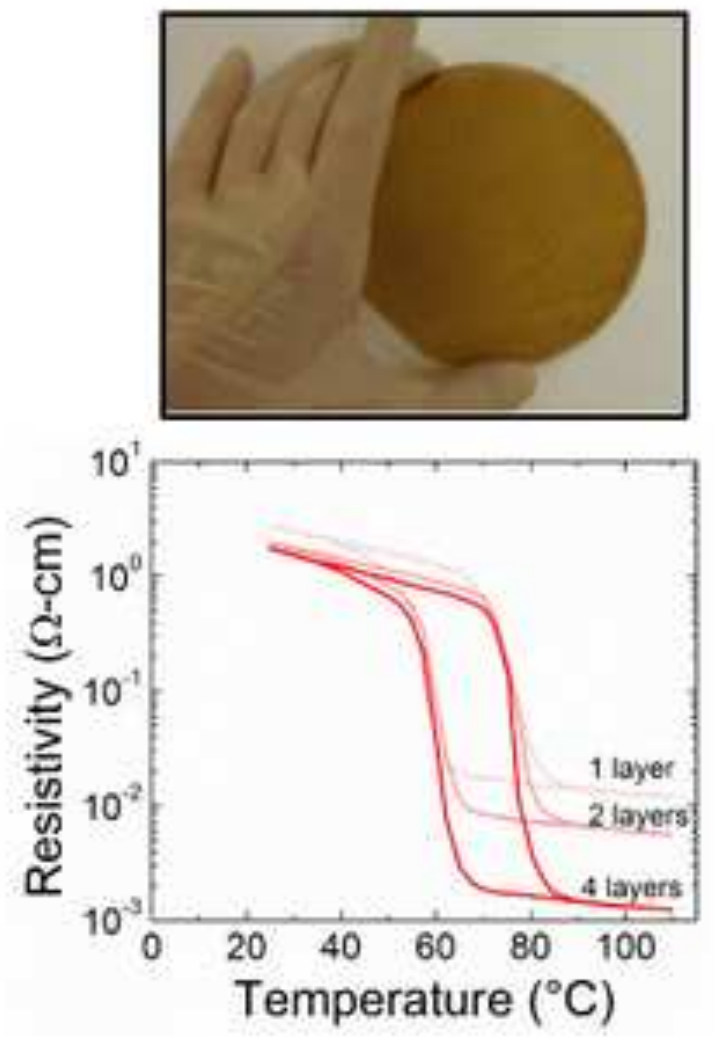

